\title{
Spectrométrie bêta appliquée aux mesures de la radioactivité dans l'environnement
}

\author{
P. BOUISSET ${ }^{1}$, R. GURRIARAN ${ }^{1}$, E. YEREBAKANIAN ${ }^{1}$, A. COURTI $^{1}$
}

(Manuscrit reçu le 18 janvier 2004, accepté le 16 août 2004)

RÉSUMÉ Les techniques employées pour quantifier les émetteurs bêta purs $\left({ }^{3} \mathrm{H},{ }^{14} \mathrm{C},{ }^{90} \mathrm{Sr}\right.$, ${ }^{36} \mathrm{Cl},{ }^{63} \mathrm{Ni}$...) dans les matrices environnementales sont basées sur une sélectivité physico-chimique de l'élément suivi d'une mesure par scintillation liquide ou comptage proportionnel. Nous avons étudié une configuration de mesure simple, basée sur des détecteurs silicium minces (PIPS), afin d'obtenir une sélectivité spectrométrique couplée ou non à une sélectivité physico-chimique. La quantification de sources pures montre que les limites de détection sont du même ordre de grandeur que celles obtenues par les méthodes conventionnelles avec l'avantage de préciser la présence d'interférents résiduels. De même, pour des mesures de surveillance ou de contrôle réglementaire, excluant une préparation chimique importante, la contribution des radionucléides naturels est souvent à la base de la mise en ouvre de mesures complémentaires. Cette contribution des naturels peut être observée afin d'exclure la nécessité de ces mesures complémentaires même si les seuils globaux réglementaires sont dépassés.

ABSTRACT Beta spectrometry applied to environmental radioactivity measurements.

Techniques used to quantify pure beta emitters $\left({ }^{3} \mathrm{H},{ }^{14} \mathrm{C},{ }^{90} \mathrm{Sr},{ }^{36} \mathrm{Cl},{ }^{63} \mathrm{Ni} \ldots\right)$ in environmental matrices are based on a physico-chemistry selection of the element followed by liquid scintillation or proportional counting. We have studied a new method using thin Si (PIPS) detectors to obtain a spectrometric discrimination with or without the physico-chimical discrimination. Detection limits for pure sources are of the same order than values obtained with standard techniques with the advantage to identify other residual radionuclides. The part of natural radionuclide is important for surveillance measurement because global results induce complementary measurements. The spectrometry technique can discriminate natural and artificial contribution without necessity of detailed measurement even results above regular thresholds.

Key words: Beta particles / environmental contamination / silicon detector / screening measurements.

\section{Introduction}

La compréhension des processus de transfert de la radioactivité dans l'environnement et la radioprotection appliquée à l'environnement et à l'homme nécessitent des moyens de mesures adaptés aux différents radionucléides. La baisse continue de la radioactivité de l'environnement, la baisse réglementaire des

\footnotetext{
1 IRSN, Laboratoire de mesure de la radioactivité de l'environnement, bâtiment 501, bois des rames, 91400 Orsay.
} 
doses acceptables, le stockage des déchets et le démantèlement à venir des installations nucléaires nécessitent une amélioration constante de ces moyens d'analyses et conjointement des expertises plus performantes. Ces besoins se traduisent en métrologie par une amélioration des limites de détection, une réduction des incertitudes, des délais d'analyses moins longs, un nombre d'analyses plus important et la quantification de tous les radionucléides, d'origine artificielle mais aussi, et de plus en plus, ceux d'origine naturelle.

Les principaux radionucléides artificiels émetteurs $\beta\left({ }^{90} \mathrm{Sr},{ }^{137} \mathrm{Cs},{ }^{134} \mathrm{Cs},{ }^{129} \mathrm{I}\right.$, ${ }^{60} \mathrm{Co}$ ) sont distribués dans l'environnement à des niveaux très faibles mais peuvent atteindre des niveaux de plusieurs dizaines de $\mathrm{Bq} / \mathrm{kg}$ en ${ }^{90} \mathrm{Sr}$ et de plusieurs milliers de $\mathrm{Bq} / \mathrm{kg}$ en ${ }^{137} \mathrm{Cs}$ en cas de situation accidentelle ou dans certaines régions déjà contaminées comme le Mercantour en France ou dans les territoires de Biélorussie. De même, les radionucléides émetteurs $\beta$ naturels sont à des niveaux variables dans l'environnement : les activités de ${ }^{40} \mathrm{~K}$ et des familles ${ }^{238} \mathrm{U}$ et ${ }^{232} \mathrm{Th}$ varient d'une centaine à quelques milliers de $\mathrm{Bq} / \mathrm{kg}$.

La détection bêta est toujours accompagnée de rayonnement gamma interférant dans le détecteur. Ce rayonnement provient aussi bien de l'échantillon lui-même que du milieu ambiant. Nous avons étudié la réponse d'un spectromètre bêta basé sur un montage en télescope ( 2 détecteurs l'un derrière l'autre) afin de discriminer par coïncidence les électrons des photons.

\section{Méthodes classiques employées pour la mesure des émetteurs $\beta$ purs}

Les méthodes de quantification des émetteurs $\beta$ sont actuellement basées sur des mesures où la discrimination est faite en amont lors de la préparation de l'échantillon avec des moyens d'extraction physico-chimique relativement long.

C'est le cas de la scintillation liquide développée depuis les années 1950 (Kallman, 1950 ; Reynolds et al., 1950) avec l'emploi d'un cocktail scintillant dont le rôle est de convertir l'énergie déposée par les électrons en photons détectés par un photomultiplicateur. Les techniques chromatographiques sont employées en amont de la mesure pour extraire et concentrer les radionucléides. La mesure simultanée des isotopes 89 et 90 du strontium dans les eaux est ainsi réalisée avec une limite de détection de 30 à $40 \mathrm{mBq} / \mathrm{l}$ pour chaque isotope.

À cause de l'interférence avec ${ }^{89} \mathrm{Sr}$, la quantification de ${ }^{90} \mathrm{Sr}$ seul a donné lieu aux développements de protocoles basés sur l'extraction du strontium et d'une phase de séparation de l'yttrium suivi de la mesure de la décroissance de ce dernier par comptage proportionnel avec des normes spécifiques par exemple pour l'eau 


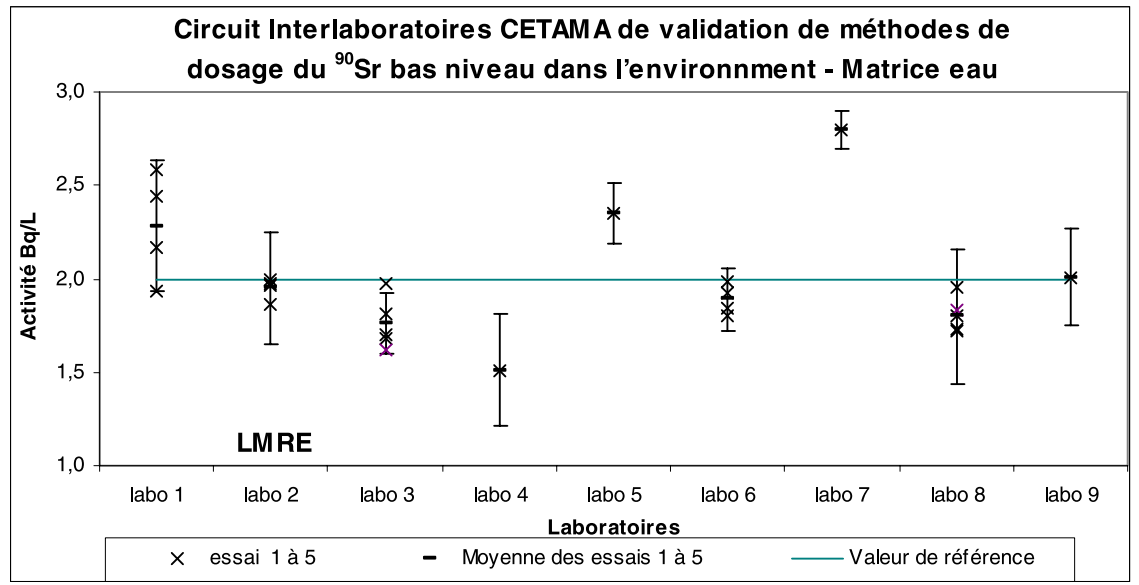

Figure 1 -Résultats du circuit interlaboratoires CETAMA de dosage du ${ }^{90} \mathrm{Sr}$ bas niveau dans l'environnement (Ansoborlo 2003).

Low level ${ }^{90} \mathrm{Sr}$ results for the intercomparison exercise organized by CETAMA.

(AFNOR, 1997) et pour les sols (AFNOR, 1999). Basés sur ces normes les protocoles radiochimiques sont adaptés aux différentes natures de matrices (en fonction de la teneur en $\mathrm{Ca}-\mathrm{Sr}$ ) et on obtient par exemple des limites de détection de $36 \mu \mathrm{Bq} / 1$ pour l'eau de mer à partir de 500 litres traités ou $0,66 \mathrm{~Bq} / \mathrm{kg}$ pour une prise d'essai de $10 \mathrm{~g}$ de sédiments (La Rosa et al., 2002).

Les circuits d'intercomparaison pour la mesure $\beta$ se limitent en général à la mesure de ${ }^{90} \mathrm{Sr}$ dans l'eau. La figure 1 montre les résultats de l'intercomparaison Cetama organisée en 2002 (Ansoborlo, 2003). Seuls 9 laboratoires français ont répondu avec des résultats peu dispersés compte tenu de l'emploi de méthodes d'extraction différentes, les mesures étant ensuite réalisées par scintillation liquide ou par comptage proportionnel.

Pour des concentrations plus élevées, le nombre de laboratoire participant est plus important comme le montre le circuit organisé en 2003 par l'IRSN dans le cadre du réseau national institué par l'article R 1333-11 du Code de la santé publique. 28 laboratoires ont proposé des résultats cohérents avec une activité moyenne de $6,19 \pm 0,48 \mathrm{~Bq} / 1$ pour une valeur de référence de $6,36 \pm 0,40 \mathrm{~Bq} / 1$ (IRSN, 2003).

En ce qui concerne les matrices solides, les intercomparaisons montrent que peu de laboratoires réalisent ces analyses à bas niveaux dans l'environnement. 
Lors de l'exercice national organisé par l'OPRI en 1998, pour du lait en poudre, 23 laboratoires ont communiqué un résultat sur 36 participants avec une valeur de référence de 30,8 $\pm 3,7 \mathrm{~Bq} / \mathrm{kg} \sec$ (OPRI, 1998).

Lorsque la concentration est plus basse, peu de laboratoires quantifient ${ }^{90} \mathrm{Sr}$. Dans le cas d'un échantillon d'algue marine par exemple proposé par le NIST où 22 laboratoires représentant 14 pays participaient, seuls 4 laboratoires ont quantifié ${ }^{90} \mathrm{Sr}$ avec une valeur inférieure à $1 \mathrm{~Bq} / \mathrm{kg} \mathrm{sec}$ (Outoula, 2003).

Pour les émissions $\beta$ de grandes énergies $\left({ }^{90} \mathrm{Y}-{ }^{90} \mathrm{Sr},{ }^{32} \mathrm{P}, \ldots\right)$, l'effet Cherenkov peut être employé en plaçant simplement l'échantillon dans de l'eau. Cette méthode est couramment utilisée pour quantifier ${ }^{90} \mathrm{Sr}$ (Randolph, 1975 ; Rucker, 1992) avec une efficacité de comptage supérieure à $40 \%$ en solution aqueuse.

Pour des radionucléides de longues périodes comme ${ }^{36} \mathrm{Cl}$ et ${ }^{14} \mathrm{C}$, les spectromètres de masses développés pour des mesures de datation sont très performants en terme de précision et de limite de détection. Dans le cas de ${ }^{36} \mathrm{Cl}$, radionucléide émis dans l'environnement lors du traitement du combustible usé, mais aussi présent naturellement dans l'environnement avec un rapport isotopique ${ }^{36} \mathrm{Cl} /{ }^{\text {total }} \mathrm{Cl}$ de l'ordre de $10^{-15}$, seul le comptage des atomes peut être employé. Les techniques radiométriques (scintillation liquide ou comptage proportionnel) sont incapables d'obtenir mieux qu'un rapport $10^{-9}$ en traitant des quantités de plusieurs dizaines de grammes avec des protocoles permettant d'éliminer les autres émetteurs $\beta$, en particulier ${ }^{129} \mathrm{I}$ (Bouisset et al., 1999). Ces grands appareillages sont très peu répandus dans le monde et ne peuvent faire face au nombre important de mesures nécessaires pour le contrôle environnemental ou pour les études radioécologiques.

\section{Méthodes spectrométriques}

Des méthodes de mesures directes ont été développées récemment pour réaliser un grand nombre de quantification de ${ }^{90} \mathrm{Sr}$ en vue d'établir un état de la contamination sur de vastes territoires suite à l'accident de Tchernobyl (Cosma, 2000). Dans ce contexte, il faut résoudre les interférences avec les autres radionucléides comme ${ }^{137} \mathrm{Cs},{ }^{106} \mathrm{Rh}, \ldots$ présents aussi dans les échantillons.

Ces méthodes sont en général basées sur des mesures par scintillation liquide ou comptage proportionnel. Une méthode de déconvolution spectrale pour la mesure simultanée de ${ }^{90} \mathrm{Sr}$ et ${ }^{89} \mathrm{Sr}$ a été proposée récemment (Heilgeist, 2000) et deux méthodes analytiques ont été développées pour des mesures multiélémentaires par scintillation liquide : la théorie de la valeur la plus probable et la déconvolution spectrale associée à une interpolation. 


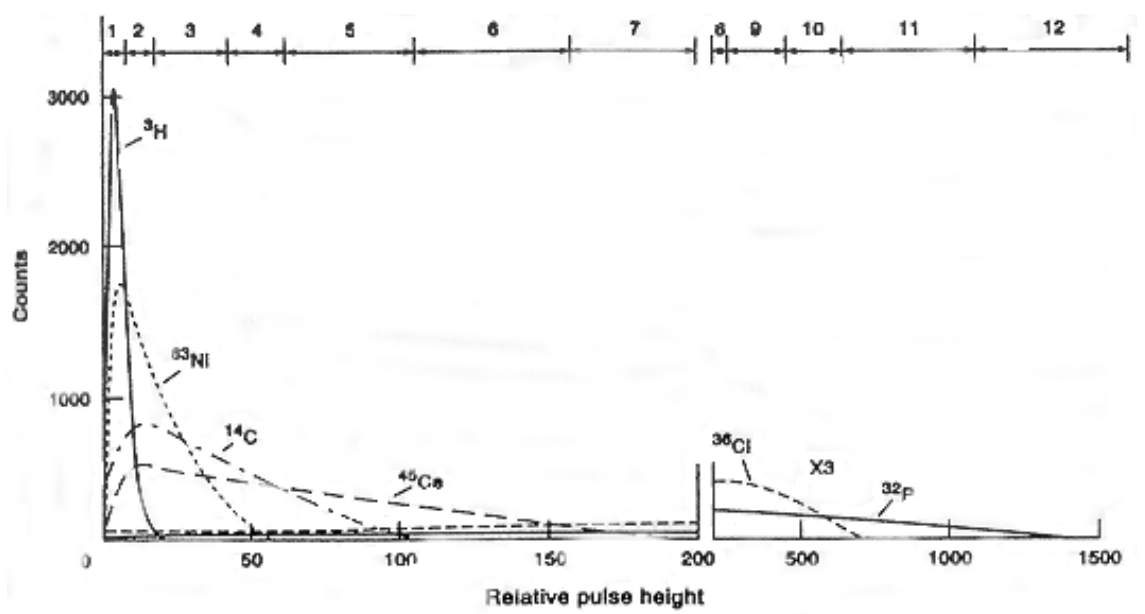

Figure 2 - Spectrogramme de 6 émetteurs $\beta$-purs (Takiue et al., 1992). 12 régions d'intérêts sont définies pour obtenir la solution la plus probable.

Liquid scintillation pulse height distributions of six pure $\beta$-emitters and region settings for analytical measurements (Takiue et al., 1992).

La théorie de la valeur la plus probable est décrite par Takiue (Takiue et al., 1991) et a été appliquée pour quantifier des mélanges de 4 radionucléides ${ }^{3} \mathrm{H},{ }^{14} \mathrm{C}$, ${ }^{32} \mathrm{P}$ et ${ }^{45} \mathrm{Ca}$ (Takiue et al., 1990) par scintillation liquide. Les moyens analytiques sont usuels, la méthode est basée sur la définition d'un nombre de régions d'intérêts supérieurs au nombre de radionucléides recherchés afin d'obtenir un système d'équations solvables. Pour chacune des régions d'intérêts, le nombre d'impulsion enregistré est la somme des impulsions de chaque radionucléide. La solution la plus probable est obtenue simplement par le calcul du déterminant du système d'équations. Les auteurs ont poursuivi cette approche pour un mélange de 6 radionucléides (Fig. 2) en définissant 12 régions d'intérêts pour résoudre le système. Pour des niveaux d'activités environnementaux, dans le cas d'un mélange de 6 radionucléides, les limites de détection sont de $10 \mathrm{~Bq} / \mathrm{l}$ à haute énergie à $50 \mathrm{~Bq} / \mathrm{l}$ à basse énergie (Takiue et al., 1992).

La méthode de déconvolution a été développée pour quantifier 2 radionucléides dont les énergies maximum sont proches comme ${ }^{35} \mathrm{~S}$ et ${ }^{14} \mathrm{C}$ (Grau Carles et al., 1993). Après une mesure par scintillation liquide, l'analyse nécessite 3 étapes :

- le spectre expérimental discret est lissé par un ajustement avec une série de Fourier ; 


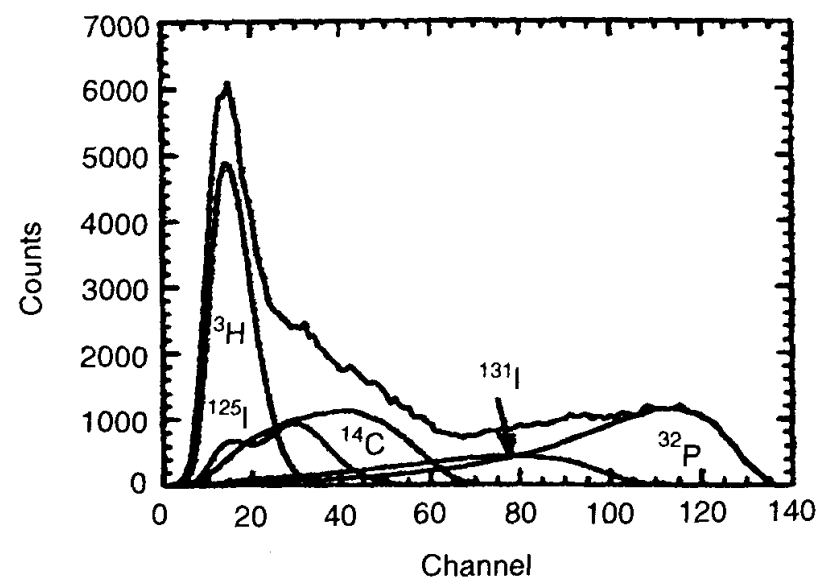

Figure 3 - Décomposition du spectre expérimental d'un mélange en 5 composantes (Grau Carles et al., 1996).

Deconvolution of a mixed spectra obtained for 5 radionuclides (Grau Carles et al., 1996).

- une déconvolution à l'aide de spectres de références ayant un quenching identique est réalisée en minimisant la relation : $\min \left\{\Sigma\left(\mathrm{y}_{\mathrm{i}}(\mathrm{X}+\mathrm{Y})-\mathrm{ay}_{\mathrm{i}}(\mathrm{X})-\right.\right.$ $\left.\left.\mathrm{by}_{\mathrm{i}}(\mathrm{Y})\right)^{2}\right\}$ où $\mathrm{y}_{\mathrm{i}}$ est le nombre de coups dans le canal i du radionucléide $\mathrm{X}$, $\mathrm{Y}$ et de la somme des 2 , a et b sont obtenus par les moindres carrés ;

- une interpolation spectrale qui a pour rôle de lisser les fluctuations statistiques.

La méthode a été étendue pour traiter le cas de plusieurs radionucléides comme le montre la figure 3 pour un mélange de 5 radionucléides (Grau Carles et al., 1996). Les résultats sont précis à $3 \%$ pour des activités de $2 \mathrm{kBq}$ en tritium et de quelques centaines de becquerel pour les autres radionucléides.

Ces méthodes n'ont pas été élaborées pour des matrices environnementales pour lesquelles une séparation partielle des radionucléides est difficilement réalisable, où les radionucléides naturels sont en quantités importantes et où l'activité des émetteurs $\beta$-purs est généralement faible par rapport à celles des autres émetteurs $\beta-\gamma$.

Pour une application de ces approches analytiques plus adaptée aux mesures environnementales, nous avons étudié un système basé sur la mesure des électrons avec des semi-conducteurs (Courti, 2002), la configuration du système pouvant être différente quand il s'agit d'une mesure en laboratoire ou d'une mesure in situ ou s'il s'agit d'une mesure de traces précises ou d'une mesure plus qualitative. 
Quelle que soit l'application envisagée, les détecteurs d'électrons sont toujours sensibles aux photons, qu'ils proviennent du milieu ambiant ou de l'échantillon, en créant eux-mêmes dans le détecteur des électrons qu'il est difficile de discriminer des électrons primaires.

\subsection{Description et principe du système de mesure}

Le système de détection employé pour cette étude est constitué :

- d'une chambre de comptage,

- de 2 détecteurs PIPS,

- d'une électronique avec analyse d'amplitude,

- d'un système informatique d'acquisition et de traitement de données.

La chambre de comptage est en acier inox de $12 \mathrm{~mm}$ d'épaisseur et de $4147 \mathrm{~cm}^{3}$ $\left(\mathrm{L} \times \mathrm{H} \times 1=180 \times 240 \times 96 \mathrm{~mm}^{3}\right)$ de volume intérieur, étanche au vide, équipée de connexions BNC.

Le spectromètre $\beta$ (Courti et al., 2002) est un télescope composé de deux détecteurs PIPS (Planar Implanted Passivited Silicon) superposés dont les avantages sont :

- résolution en énergie de l'ordre de $15 \mathrm{keV}$,

- grande surface active pour optimiser l'angle solide de détection,

- faible épaisseur pour minimiser l'interaction des photons associée avec une mesure en anticoïncidence pour les rejeter lorsqu'ils interagissent,

- utilisation simple (fonctionnant à température ambiante),

- éprouvé pour d'autres applications de mesure (spectrométrie $\alpha$ en routine), et

- faible coût et encombrement.

Le détecteur D1 (300 $\mu \mathrm{m}$ d'épaisseur et $300 \mathrm{~mm}^{2}$ de surface active) le plus proche de l'échantillon est sur une monture annulaire. Le détecteur D2 $(500 \mu \mathrm{m}$, $450 \mathrm{~mm}^{2}$ ) est sur une monture axiale. Les montures annulaire et axiale, en aluminium, ont une prise de type microdot sur le côté pour D1 et sur le haut et dans son axe pour D2 (Fig. 4).

Le principe de ce montage en télescope est basé sur les modes d'interactions primaires différents entre les photons et les électrons. Les électrons ralentissent de façon continue dans le détecteur: ils sont donc toujours détecté dans les deux détecteurs s'ils ont une énergie suffisante. Les photons interagissent par effet Compton principalement uniquement dans l'un ou l'autre des détecteurs mais pas dans les deux à la fois. Ainsi, une coïncidence entre les 2 détecteurs permet de rejeter tous les signaux provenant des photons. 


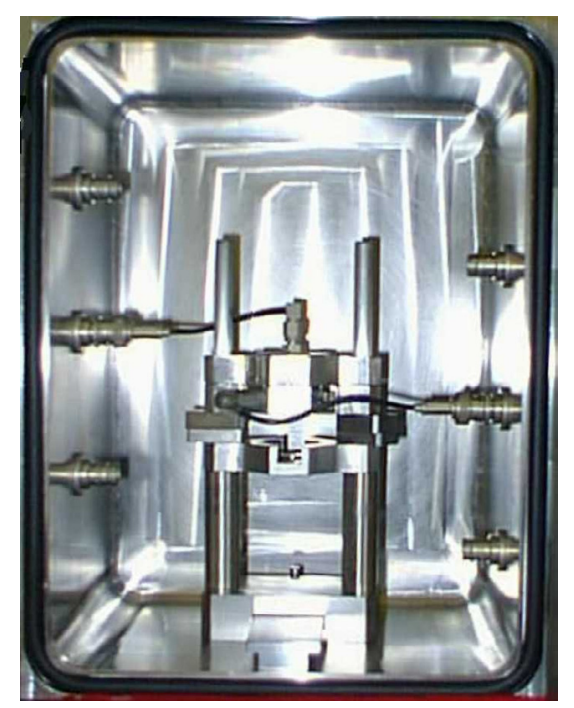

Figure 4-Vue de l'intérieur de la chambre de comptage.

Inside view of the counting chamber.

\section{2. Étalonnage des détecteurs}

L'étalonnage en énergie a été réalisé avec les électrons de conversions d'une source de ${ }^{207} \mathrm{Bi}$. En accord avec les calculs de J.M. Berger en 1969 (Berger et al., 1969), nous avons en effet observé que $20 \%$ des électrons à $480 \mathrm{keV}$ et plus de $0,5 \%$ à $1050 \mathrm{keV}$ étaient totalement arrêtés dans le détecteur de $300 \mu \mathrm{m}$ seulement d'épaisseur (phénomène de diffusion). Le résultat d'un calcul de type Monte Carlo avec le code GÉANT ${ }^{2}$ est superposé au spectre expérimental (Fig. 5) d'une source de ${ }^{207} \mathrm{Bi}$ de $37 \mathrm{kBq}$ (activité au 7 avril 2000). La position des pics est confirmée par le calcul, les pics somme sont identifiés et une structure plus fine apparaît car notre simulation n'intègre pas la résolution de l'ensemble de mesure.

\subsection{Résultats expérimentaux}

\subsubsection{Sources radioactives pures}

Des spectres expérimentaux de sources $\beta$-pures et $\beta-\gamma$ ont été réalisés pour mettre en évidence entre autres les régions spectrales caractéristiques et déterminer les contributions gamma de chaque radionucléide à l'aide du montage en coïncidence (Courti, 2002).

\footnotetext{
2 GÉANT: Detector description and simulation tool, CERN Program Library W2013, CERN, Geneva.
} 


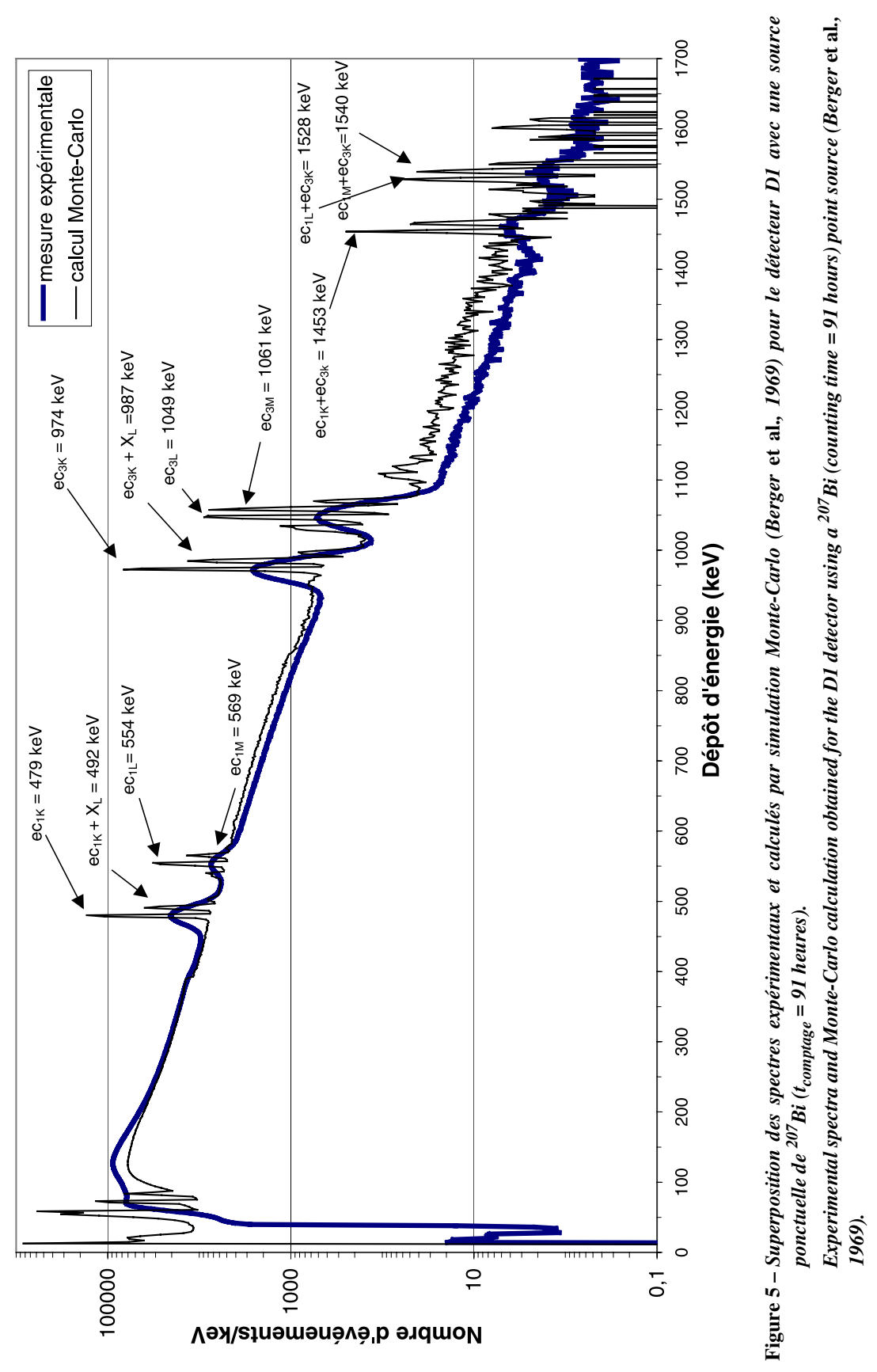

RADIOPROTECTION - VOL. $40-\mathrm{N}^{\circ} 1(2005)$

37 


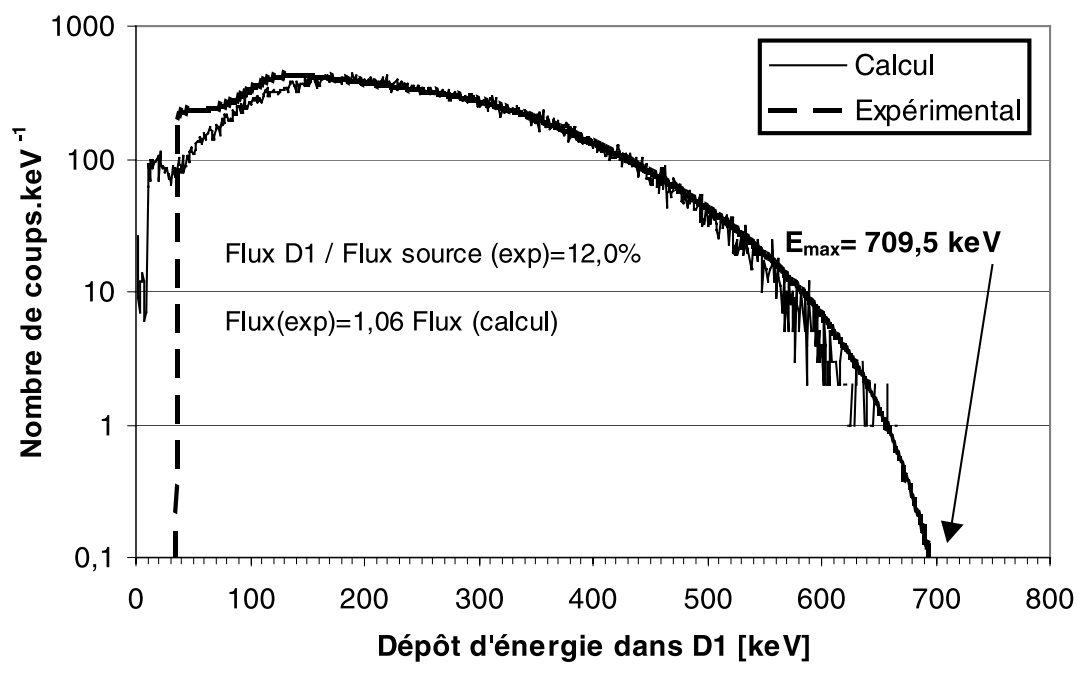

Figure 6 - Spectre de dépôt d'énergie de ${ }^{36} \mathrm{Cl}(4000 \mathrm{~Bq})$ expérimental et calculé avec le code GÉANT dans D1.

Experimental energy spectra of ${ }^{36} \mathrm{Cl}(4000 \mathrm{~Bq})$ source compared with a Monte-Carlo calculation using GÉANT Code for the D1 detector.

La figure 6 montre le spectre des dépôts d'énergie d'une source de ${ }^{36} \mathrm{Cl}$ vu par D1 comparé au calcul GÉANT. Avec une énergie moyenne de $250 \mathrm{keV}$, seuls $12 \%$ des électrons émis par la source sont détectés par D1 et moins de $1 \%$ sont détectés dans D2.

La détection de ${ }^{36} \mathrm{Cl}$ est située dans la région d'énergie inférieure à $700 \mathrm{keV}$ pour D1 et inférieure à $600 \mathrm{keV}$ pour D2. Les événements détectés sur D1 se répartissent à $89 \%$ dans la voie en anticoïncidence et seulement à $11 \%$ sur la voie en coïncidence car le spectre émis est de faible énergie.

La voie D1 en mode anticoïncidence dans la gamme d'énergie de 70 à $700 \mathrm{keV}$ permet d'atteindre une limite de détection de $0,026 \mathrm{~Bq}$ dans la source en 60 heures de comptage et près de $0,2 \mathrm{~Bq}$ en 1 heure dans les conditions de ces mesures sans que le mouvement propre soit optimisé (en plaçant par exemple un blindage de plomb autour de la chambre de comptage).

Dans le cas d'une source de ${ }^{137} \mathrm{Cs}$ de $20 \mathrm{kBq}$, le radionucléide est caractérisé par ses pics de conversion interne à $624 \mathrm{keV}, 657 \mathrm{keV}$, ses deux énergies maximales à 511,5 et $1173 \mathrm{keV}$, et la forme du spectre d'impulsion obtenue. Les nombres d'impulsions pour D1, D2 et celles de D2 en coïncidence avec D1 sont indiqués sur la figure 7 . 
SPECTROMÉTRIE BÊTA APPLIQUÉE AUX MESURES DE LA RADIOACTIVITÉ

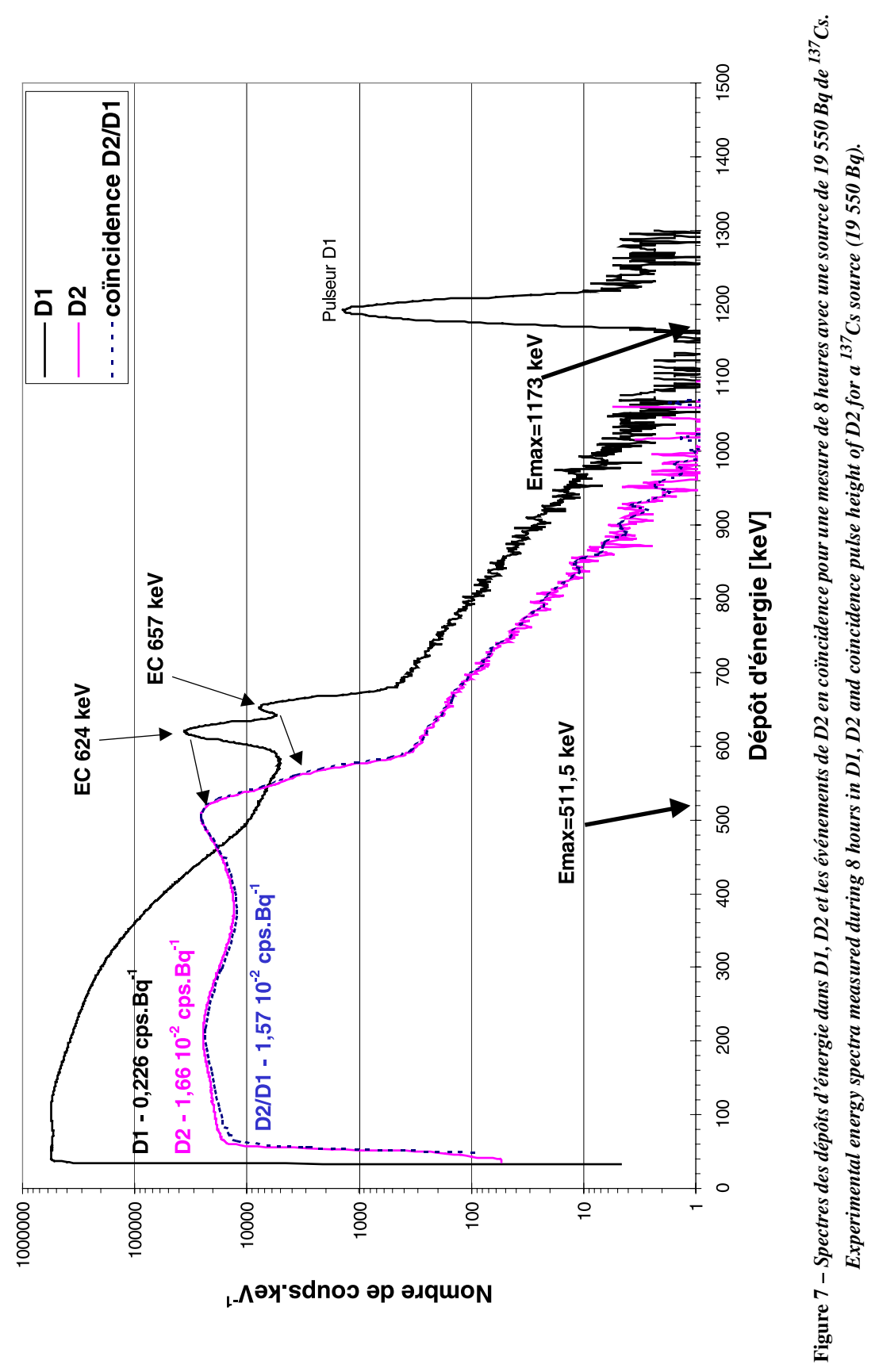

RADIOPROTECTION - VOL. 40 - $\mathrm{N}^{\circ} 1(2005)$

39 
TABLEAU I

Caractéristiques expérimentales des différents radionucléides étudiés avec les détecteurs D1 et D2 (Courti, 2002).

Characteristic data for experimental source measurements for D1 and D2 detectors (Courti, 2002).

\begin{tabular}{|c|c|c|c|c|c|c|c|c|c|}
\hline \multirow[b]{2}{*}{ Source } & \multirow[b]{2}{*}{$\begin{array}{l}\text { Diamètre } \\
{[\mathrm{mm}]}\end{array}$} & \multirow[b]{2}{*}{$\begin{array}{l}\mathrm{E}_{\max } \\
{[\mathrm{keV}]}\end{array}$} & \multirow[b]{2}{*}{$\begin{array}{c}\mathrm{EC} \\
{[\mathrm{keV}]}\end{array}$} & \multicolumn{6}{|c|}{ Intervalle $40-1100 \mathrm{keV}$} \\
\hline & & & & $\begin{array}{c}\varepsilon_{1} \Omega_{1}(\mathrm{D} 1) \\
{[\%]}\end{array}$ & $\begin{array}{c}\varepsilon_{2} \Omega_{2}(\mathrm{D} 2) \\
{[\%]}\end{array}$ & $\begin{array}{c}\text { D2/D1 } \\
{[\%]}\end{array}$ & $\begin{array}{c}\text { C/D2 } \\
{[\%]}\end{array}$ & $\begin{array}{c}\text { Taux } \gamma \text { D1 } \\
{[\%]}\end{array}$ & $\begin{array}{c}\text { Taux } \gamma \text { D2 } \\
{[\%]}\end{array}$ \\
\hline${ }^{241} \mathrm{Am}$ & 3 & - & - & 0,37 & 0,02 & 10 & 0,9 & 12,8 & 99,1 \\
\hline${ }^{109} \mathrm{Cd}$ & 3 & - & 87,3 & 0,039 & 0,003 & 7,3 & 0,0 & 9,9 & 100 \\
\hline${ }^{207} \mathrm{Bi}$ & 3 & - & $\begin{array}{c}481,7 \\
553,8 \\
975,6 \\
1051\end{array}$ & 42,2 & 17,3 & 35,2 & 84,6 & 7,3 & 15,4 \\
\hline${ }^{36} \mathrm{Cl}$ & 6 & 709 & - & 12,7 & 0,5 & 10,7 & 100,0 & 0,0 & 0,0 \\
\hline${ }^{90} \mathrm{Sr}-{ }^{90} \mathrm{Y}$ & 3 & 2284 & - & 40,8 & 8,3 & 19,6 & 99,3 & 0,2 & 0,7 \\
\hline${ }^{137} \mathrm{Cs}$ & 3 & 1173,2 & $\begin{array}{c}624,2 \\
657\end{array}$ & 22,6 & 1,7 & 7,4 & 95,0 & 0,5 & 5,0 \\
\hline${ }^{134} \mathrm{Cs}$ & 3 & $\begin{array}{l}415,1 \\
657,8\end{array}$ & $\begin{array}{c}567,25 \\
758,4\end{array}$ & 40,5 & 3,0 & 7,5 & 82,7 & 1,7 & 17,3 \\
\hline${ }^{60} \mathrm{Co}$ & 3 & $\begin{array}{c}317,9 \\
1491,1\end{array}$ & - & 16,0 & 3,2 & 4,0 & 4,7 & 0,5 & 52,6 \\
\hline $\begin{array}{c}\text { Mt propre } \\
(60 \mathrm{~h})\end{array}$ & $\begin{array}{c}\text { '3' } \\
\text { pour calcul } \\
\text { taux } \gamma\end{array}$ & - & - & - & - & 143 & 28,4 & 137 & 72 \\
\hline
\end{tabular}

La résolution des pics des électrons de conversion est plus dégradée dans D2 et décalée vers les basses énergies de $110 \mathrm{keV}$ suite à la traversée de $300 \mu \mathrm{m}$ de silicium (D1).

Tous les électrons reçus par D2 doivent être en coïncidence avec un signal de D1 puisque l'angle solide de D1 est plus grand que celui de D2. Mouvement propre soustrait, les évènements qui ne sont pas en coïncidence dans D2 sont des photons, d'où le nombre de photons dans D1 compte tenu des angles solides des 2 détecteurs et de leurs masses de silicium.

Le taux $\gamma$ dans les détecteurs D1 et D2, le taux de coïncidence $\mathrm{C}$ par rapport à D2 et le taux d'événements relatifs D2/D1 sont indiqués dans le tableau I pour plusieurs radionucléides. Sont aussi indiqués dans ce tableau quelques données spectrométriques importantes et les rendements de détection $\varepsilon \Omega$ (efficacité et angle solide) calculés à partir du nombre d'événements détectés par rapport au nombre d'électrons émis par la source ( $\varepsilon$ tend vers 1 pour les électrons de grande énergie). Par rapport aux approches analytiques discutées précédemment, nous disposons d'un système d'équation supplémentaire pour identifier et quantifier les 
TABLEAU II

Rapport des activités de ${ }^{137} \mathrm{Cs}$ entre les sols Biélorusses comparés aux rapports des aires mesurées sur D1, D2 et en cö̈ncidence entre 100 et $900 \mathrm{keV}$.

${ }^{137}$ Cs activity ratio of Belarus soils compared to the measured area in D1, D2 and for the coincidence spectra between 100 and $900 \mathrm{keV}$.

\begin{tabular}{cccc}
\hline & Biélo16/Biélo2,2 & Biélo122/Biélo2,2 & Biélo122/Biélo16 \\
\hline Rapport activités & 7,4 & 55,5 & 7,5 \\
\hline Rapport aires D1 & 6,6 & 45,7 & 6,9 \\
Rapport aires D2 & 8,5 & 72,6 & 8,5 \\
Rapport aires en coïncidence & 7,5 & 56,1 & 7,5 \\
\hline
\end{tabular}

radionucléides dans un mélange ou pour mettre simplement en évidence la présence d'un interférant.

\subsubsection{Applications à la mesure directe d'échantillons contaminés en ${ }^{137} \mathrm{Cs}$}

Trois échantillons de sols prélevés dans les régions de Biélorussie contaminées lors de l'accident de Tchernobyl en 1986 ont été mesurés directement (Yerebakanian, 2003) après avoir été simplement séchés et analysés préalablement par spectrométrie gamma. Les spectres obtenus dans D1 sont reportés en figure 8 ainsi que le spectre correspondant à une source ponctuelle de ${ }^{137} \mathrm{Cs}$. Pour ces mesures, les échantillons sont placés dans un porte échantillon en Téflon évidé de $10 \mathrm{~mm}$ de diamètre et de $5 \mathrm{~mm}$ de profondeur et la chambre de mesure reste à pression atmosphérique. Les concentrations en radionucléides naturels $\left({ }^{40} \mathrm{~K}\right.$ et familles radioactives du thorium et de l'uranium) sont du même ordre de grandeur pour tous les échantillons, environ $130 \mathrm{~Bq} / \mathrm{kg}$ et $10 \mathrm{~Bq} / \mathrm{kg}$ respectivement. La présence des électrons de conversion de ${ }^{137} \mathrm{Cs}$ n'apparaît que sur l'échantillon le plus actif à $122 \mathrm{kBq} / \mathrm{kg}$.

Les rapports d'activités en ${ }^{137} \mathrm{Cs}$ entre ces 3 échantillons sont comparés aux rapports des aires mesurées entre $100 \mathrm{keV}$ et $900 \mathrm{keV}$ dans le tableau II. Ces données correspondent aux aires brutes auxquelles a été soustrait le mouvement propre. Par contre, ${ }^{40} \mathrm{~K}$ et U-Th ne sont pas pris en compte. On constate sur D1 et D2 que la proportionnalité n'est pas observée entre les activités de ${ }^{137} \mathrm{Cs}$ et le nombre d'événement enregistré avec les PIPS : près de $20 \%$ d'écart au maximum. Par contre les résultats sont concordants à prés de $1 \%$ sur les valeurs en coïncidence car l'interaction des photons est alors rejetée. Un facteur d'étalonnage peut donc en être déduit afin de réaliser une déconvolution spectrale pour une identification et une quantification des radionucléides.

Des spectres de références ont été établis pour ${ }^{40} \mathrm{~K}$, les familles ${ }^{238} \mathrm{U}+{ }^{235} \mathrm{U}+{ }^{232} \mathrm{Th}$ (activité de ${ }^{238} \mathrm{U}$ et ${ }^{232} \mathrm{Th}$ égale et ${ }^{235} \mathrm{U}$ en rapport naturel) et 


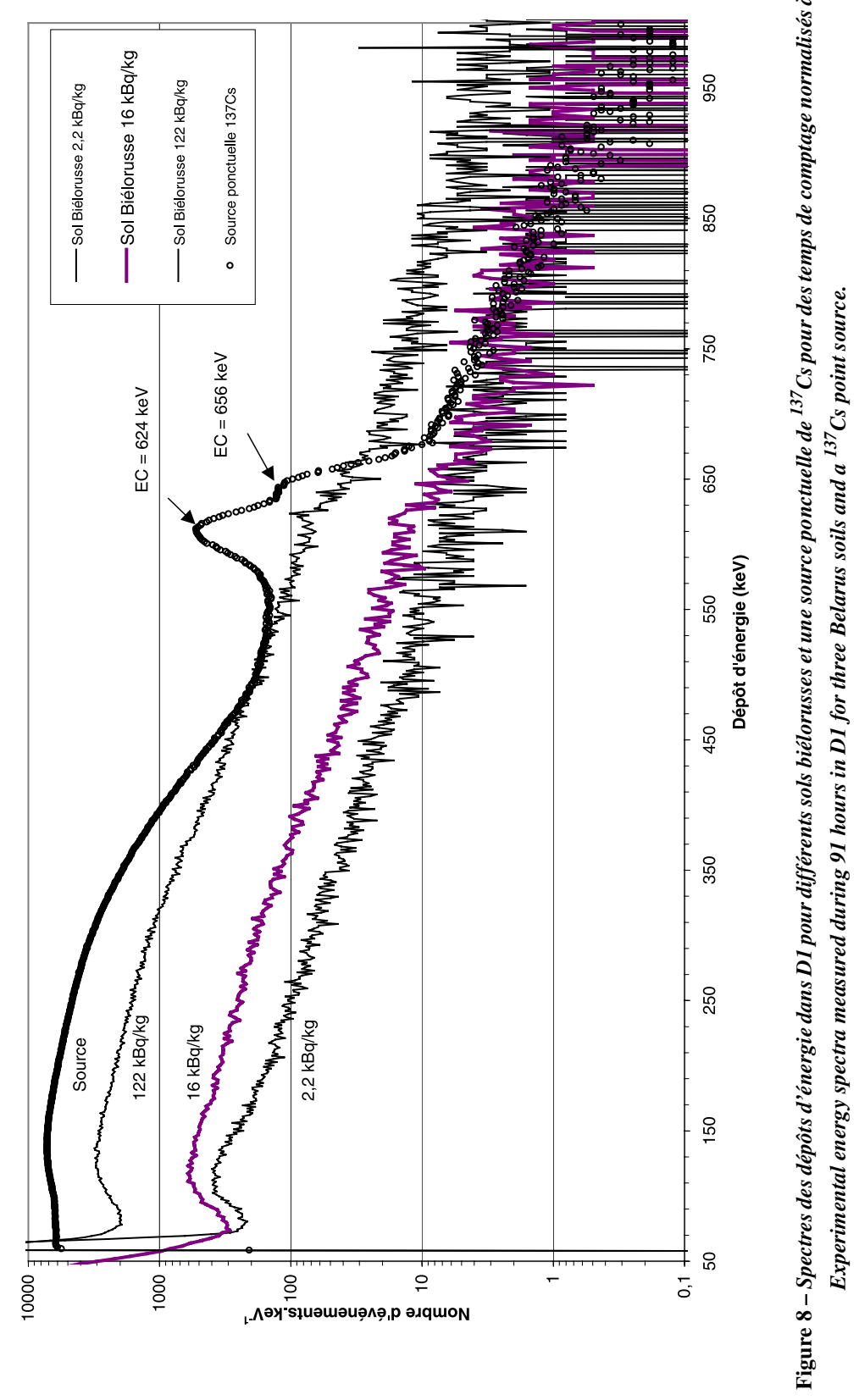




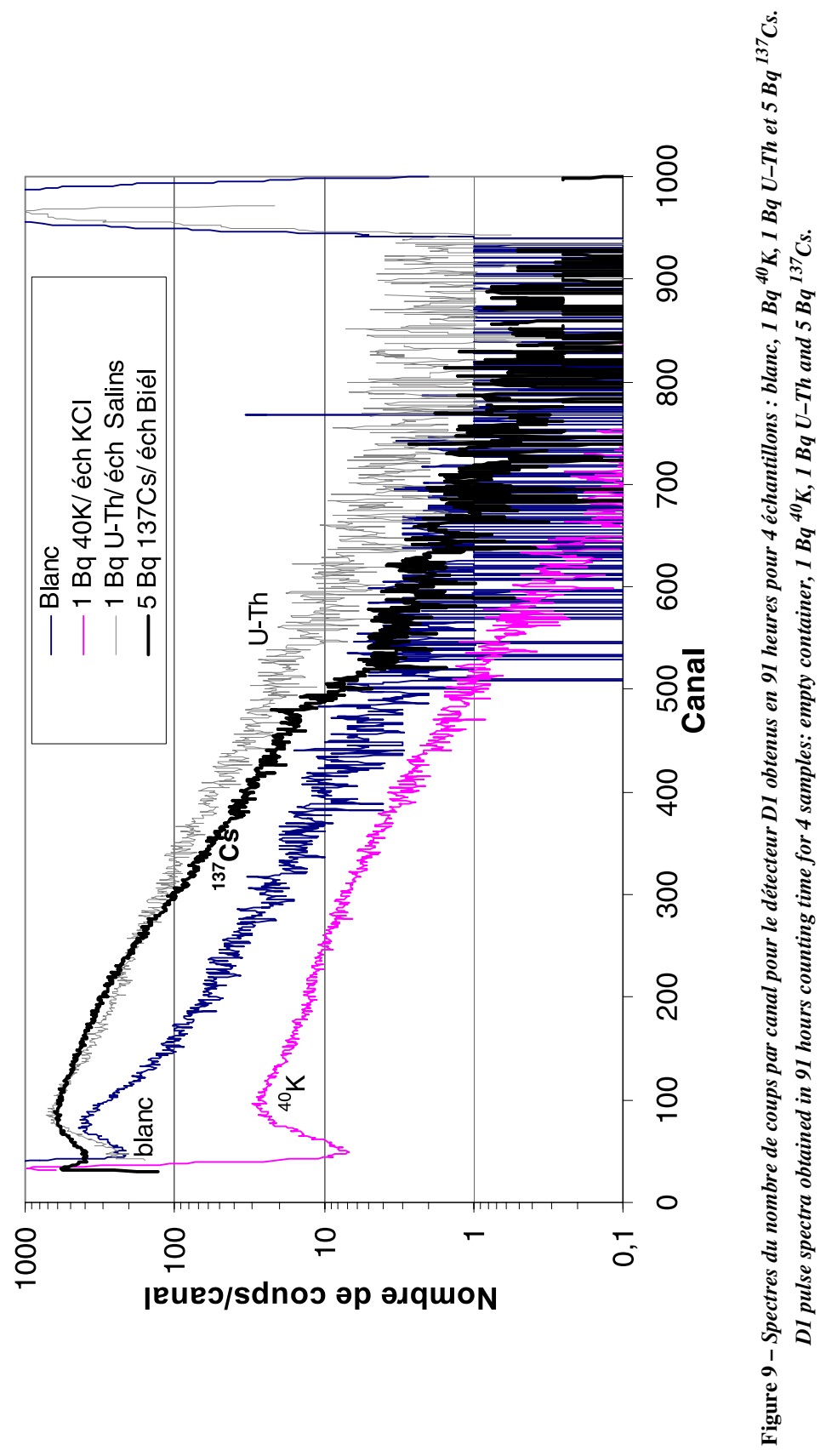


du ${ }^{137}$ Cs. Les spectres obtenus sur le détecteur D1 pour ces radionucléides et un spectre de blanc sont superposés figure 9. Ces données correspondent aux mesures brutes auxquelles le blanc est soustrait pour les 3 échantillons, ${ }^{40} \mathrm{~K}$ est soustrait du sol biélorusse et du sable des Salins et le spectre des familles U-Th est soustrait pour la mesure du sol biélorusse uniquement. Les activités des échantillons sont suffisamment élevées en ${ }^{40} \mathrm{~K}(16,4 \mathrm{kBq} / \mathrm{kg})$ en U-Th $(3 \mathrm{kBq} / \mathrm{kg})$ et en ${ }^{137} \mathrm{Cs}$ $(122 \mathrm{kBq} / \mathrm{kg})$ respectivement dans les 3 échantillons pour que les soustractions successives des autres composantes (blanc, ${ }^{40} \mathrm{~K}$ et U-Th) affectent peu le résultat.

\section{Conclusion}

Une spectrométrie $\beta$ pour les matrices environnementales est développée afin de compléter la sélectivité radiochimique employée actuellement pour la mesure des traces. La configuration de mesure étudiée, basée sur des détecteurs PIPS en montage télescopique, permet de compléter l'expertise par la reconnaissance d'interférents résiduels après radiochimie sélective ou en l'associant à des méthodes d'analyses développées pour la scintillation liquide (valeur la plus probable ou recouvrement spectral), en apportant une meilleure résolution et à l'aide d'un système de coïncidence permettant d'obtenir une distribution des activités sur un mélange inconnu.

Des spectres de références pour des sources pures $\left({ }^{241} \mathrm{Am},{ }^{109} \mathrm{Cd},{ }^{207} \mathrm{Bi},{ }^{36} \mathrm{Cl}\right.$, ${ }^{90} \mathrm{Sr}-{ }^{90} \mathrm{Y},{ }^{137} \mathrm{Cs},{ }^{134} \mathrm{Cs}$ et ${ }^{60} \mathrm{Co}$ ) ont été établis ainsi que la fonction de réponse de notre système expérimental en coïncidence pour chacune de ces sources.

Pour une application à la mesure directe d'échantillons de l'environnement, des spectres de référence des radionucléides naturels $\left({ }^{40} \mathrm{~K}\right.$ et famille radioactive du thorium + uranium) ont été réalisés dans une géométrie de mesure réaliste pour cette application. L'interférence des photons peut être résolue par un simple montage en coïncidence. La quantification des radionucléides détectés doit être apportée par déconvolution spectrale sur la base d'une bibliothèque de spectres de référence en cours d'établissement.

\section{RÉFÉRENCES}

AFNOR (1997) norme NF M 60-800.

AFNOR (1999) norme NF M 60-790-7.

Ansoborlo E. (2003) Circuit interlaboratoires de validation de dosage du ${ }^{90} \mathrm{Sr}$ bas niveau dans l'environnement, Note Technique DRPT/CETAMA/2003/05, 19 p.

Berger M.J., Seltzer S.M., Chappell S.E., Humphreys J.C., Motz J.W. (1969) Response of silicon detectors to monoenergetic electrons with energies between 0.15 and $5.0 \mathrm{MeV}$, Nucl. Instrum. Meth. 69, 181-193. 
Bouisset P., Goutelard F., Fournier M., Sharma P., Maro D., Calmet D (1999) Mesurage de ${ }^{36} \mathrm{Cl}$. Détermination de l'impact de l'usine de la Hague dans l'environnement terrestre. In : Journées Technique de la CETAMA, 14-15 septembre 1999, Aix-en-Provence.

Cosma C. (2000) Stontium-90 measurement after the Chernobyl accident in Tomanian samples without chemical separation, Spectrochim. Acta B 55, 1165-1171.

Courti A. (2002) Étude de la fonction de réponse d'une chaîne de mesure constituée de détecteurs silicium minces en vue d'une application à la mesure d'émetteurs $\beta$ et $\beta-\gamma$ dans des échantillons de l'environnement, Thèse, université Paris-XI, 138 p.

Courti A., Bouisset P., Chevallier P. (2002) Beta spectrometry for environmental radioactivity measurements. In: Ecorad 2001, 3-7 September 2001, Aix-en-Provence, numéro spécial de Radioprotection 37 C1 (F. Bréchignac, Ed.) pp. 911-916. EDP Sciences, Les Ulis.

Grau Carles A., Rodriguez Barquero L, Grau Malonda A. (1993) Standardization of ${ }^{14} \mathrm{C}$ and ${ }^{35} \mathrm{~S}$ mixtures, Nucl. Instrum. Meth. A 235, 34-240.

Grau Carles A., Grau Malonda A., Gomez Gil V. (1996) Standardization of $\mathrm{U}\left(\mathrm{X}_{1}+\mathrm{X}_{2}\right)$ : The ${ }^{234} \mathrm{Th}+$ ${ }^{234 \mathrm{~m}} \mathrm{~Pa}+{ }^{230} \mathrm{Th}$ mixture, Nucl. Instrum. Meth. A 369, 431-436.

Heilgeist M. (2000) Use of extraction chromatography, ion chromatography and liquid scintillation spectrometry for rapid determination of strontium- 89 and strontium- 90 in food in cases of increased release of radionuclides, J. Radioanal. Nucl. Chem. 245, 249-254.

IRSN (2003) Rapport : Résultats de l'intercomparaison 71SH300, 8 p.

Kallman H. (1950) Scintillation counting with solutions, Phys. Rev. 78, 621-622.

La Rosa J., Oregioni B., Liong Wee Kwong L., Lee S.H., Gastaud J., Levy I., Povinec P. (2002) Approaches to ${ }^{90} \mathrm{Sr}$ determination in marine environmental materials. In: Ecorad 2001, 3-7 September 2001, Aix-en-Provence, numéro spécial de Radioprotection 37 C1 (F. Bréchignac, Ed.) pp. 921-925. EDP Sciences, Les Ulis.

OPRI (1998) Rapport : Résultats concernant l'intercomparaison organisée par l'OPRI - Échantillon $\mathrm{n}^{\circ} 64 \mathrm{~L} 300,10 \mathrm{p}$

Outoula I. (2003) NIST - Seaweed SRM preliminary results. Communication privée.

Randolph R.B. (1975) Determination of strontium-09 and strontium-89 by Cerenkov and liquidscintillation counting, Appl. Radiat. Isotop. 26, 9-16.

Reynolds G.T., Harrison F.B., Salvini G. (1950) Liquid scintillation counters, Phys. Rev. 78, 488.

Rucker T.L. (1992) Calculational method for the resolution of ${ }^{90} \mathrm{Sr}$ and ${ }^{89} \mathrm{Sr}$ counts from Cherenkov and liquid scintillation counting. In: Liquid Scintillation Counting and Organic Scintillators $(\mathrm{H}$. Ross, J.E. Noakes, J.D. Spaulding, Eds.) pp. 529-535. Lewis Publishers, Chealsea MI.

Takiue M., Matsui Y., Natake T., Yoshizawa Y. (1990) A new approach to analytical radioassay of multiple beta-labeled samples using a liquid scintillation spectrometer, Nucl. Instrum. Meth. A 293, 596-600.

Takiue M., Matsui Y., Fujii H. (1991) Liquid scintillation radioassay for multiple radionuclide mixtures by the most probable value theory, J. Radioanal. Nucl. Chem. 152, 227-236.

Takiue M., Matsui Y., Natake T., Fujii H. (1992) Nuclide identification of pure-beta emitter mixtures with liquid scintillation spectrometry, Appl. Radiat. Isot. 43, 853-857.

Yerebakanian E. (2003) Spectrométrie Bêta appliquée à des échantillons à l'état solide de l'environnement. Rapport LMRE 2003-19, 45 p. + annexes. Non publié. 